\title{
A long terminal repeat retrotransposon of Schizosaccharomyces japonicus integrates upstream of RNA pol III transcribed genes

Yabin Guo ${ }^{2 \dagger}$, Parmit Kumar Singh ${ }^{1 \dagger}$ and Henry L. Levin ${ }^{1 *}$

\begin{abstract}
Background: Transposable elements (TEs) are common constituents of centromeres. However, it is not known what causes this relationship. Schizosaccharomyces japonicus contains 10 families of Long Terminal Repeat (LTR)-retrotransposons and these elements cluster in centromeres and telomeres. In the related yeast, Schizosaccharomyces pombe LTR-retrotransposons Tf1 and Tf2 are distributed in the promoter regions of RNA pol II transcribed genes. Sequence analysis of TEs indicates that Tj1 of S. japonicus is related to Tf1 and Tf2, and uses the same mechanism of self-primed reverse transcription. Thus, we wondered why these related retrotransposons localized in different regions of the genome.

Results: To characterize the integration behavior of Tj1 we expressed it in S. pombe. We found Tj1 was active and capable of generating de novo integration in the chromosomes of $\mathrm{S}$. pombe. The expression of $\mathrm{Tj} 1$ is similar to Type $\mathrm{C}$ retroviruses in that a stop codon at the end of Gag must be present for efficient integration. 17 inserts were sequenced, 13 occurred within 12 bp upstream of tRNA genes and 3 occurred at other RNA pol III transcribed genes. The link between Tj1 integration and RNA pol III transcription is reminiscent of Ty3, an LTR-retrotransposon of Saccharomyces cerevisiae that interacts with TFIIIB and integrates upstream of tRNA genes.
\end{abstract}

Conclusion: The integration of Tj1 upstream of tRNA genes and the centromeric clustering of tRNA genes in S. japonicus demonstrate that the clustering of this TE in centromere sequences is due to a unique pattern of integration.

Keywords: Tj1, Long terminal repeat retrotransposon, Schizosaccharomyces japonicus, Schizosaccharomyces pombe, Integration

\section{Background}

Centromeres contain a complex range of repeat DNA that typically contains clusters of transposable elements and repeat sequences derived from transposable elements [1-10]. The repeat nature of centromeres plays an important role in the assembly of heterochromatin and the formation of higher order structure that interacts with microtubules to mediate chromosome segregation $[9,11]$. Although the clustering of transposable elements in and around centromeres occurs widely in eukaryotes from fungi to humans, it is not clear whether this relationship is

\footnotetext{
* Correspondence: levinh@mail.nih.gov

${ }^{\dagger}$ Equal contributors

'Section on Eukaryotic Transposable Elements, Program in Cellular Regulation and Metabolism, Eunice Kennedy Shriver National Institute of

Child Health and Human Development, National Institutes of Health, Building

18 T, room 106, Bethesda, MD 20892, USA

Full list of author information is available at the end of the article
}

due to the integration preferences of transposable elements or the accumulation of transposable elements in centromeres because there is little selection against them.

Whole genome sequencing of the fission yeast Schizosaccharomyces japonicus showed large numbers of transposable elements in the centromeres of all three chromosomes (Fig. 1a) [6]. Closer inspection of the centromere sequences showed clusters of tRNA genes were interspersed between the transposable elements (Fig. 1b) [6]. Analysis of the $S$. japonicus genome identified 13 full length long terminal repeat (LTR)-retrotransposons and 251 fragments of LTRretrotransposons that together comprise $309 \mathrm{~kb}$ or $2.6 \%$ of the $11.7 \mathrm{Mb}$ genome of S. japonicus [6]. Phylogenetic analysis revealed that these transposable element sequences are derived from 10 distinct families of LTRretrotransposons named $\mathrm{Tj} 1$ through $\mathrm{Tj} 10$ that are all members of the gypsy/Ty3 group of LTR-retrotransposons. One of the full-length LTR-retrotransposons belongs to the 


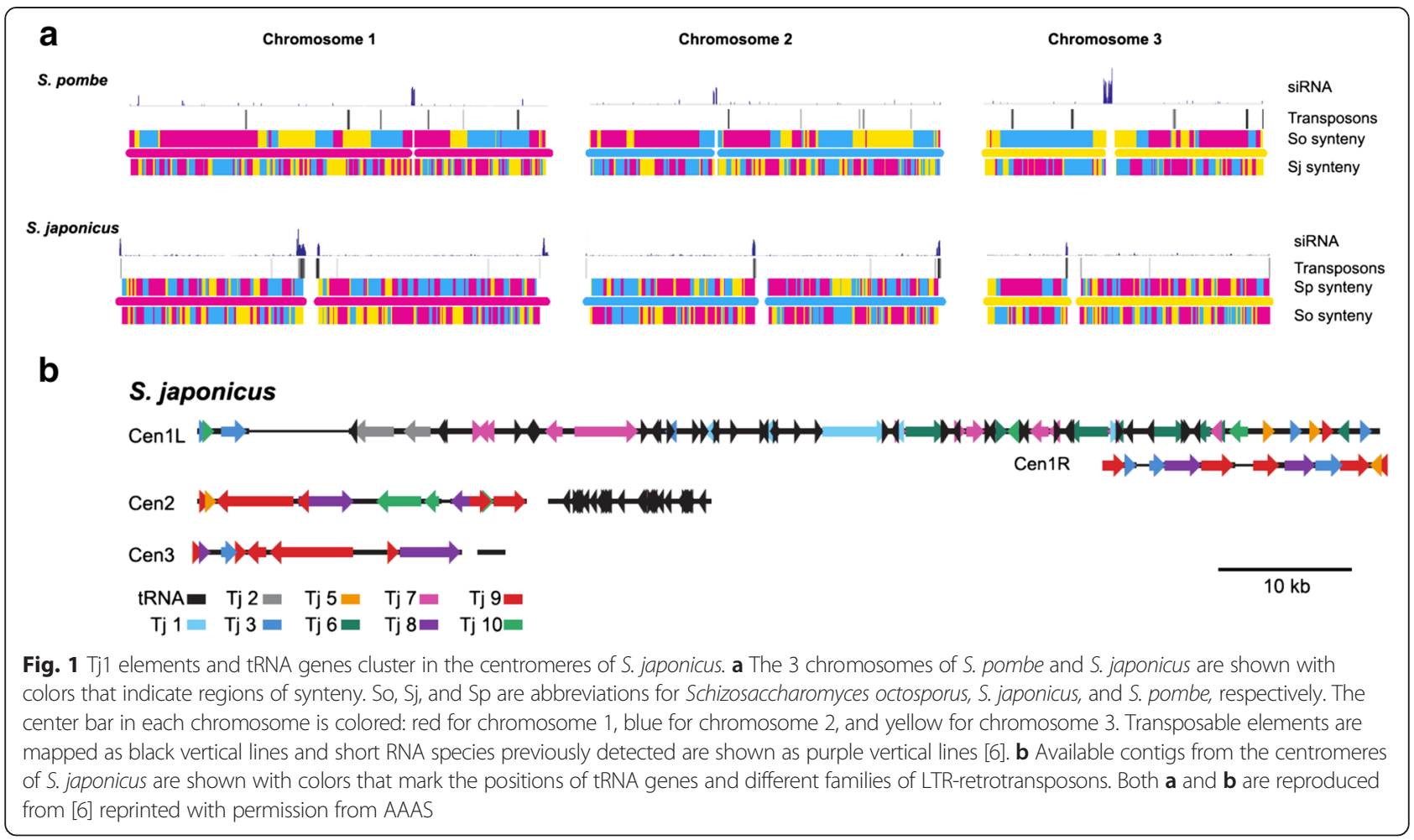

Tj1 family, is 5,003 bp in length, has two identical LTRs of $244 \mathrm{bp}$, has intact Gag and Pol open reading frames, and has an intact target site duplication of $5 \mathrm{bp}$ on either side of the element (Fig. 2a). These properties suggest that this copy of $\mathrm{Tj} 1$ may be functional. Interestingly, the ORF for Gag possesses a stop codon upstream of Pol and the Pol ORF lacks an AUG codon. This configuration occurs in the type $C$ retroviruses and functions as an unusual form of translational regulation that generates low levels of Pol proteins relative to Gag [12].

Another unusual property of $\mathrm{Tj} 1$ is the sequence of the primer-binding site (PBS) is complementary to the $5^{\prime}$ end of the Tj1 mRNA suggesting that Tj1 uses self-priming to initiate reverse transcription (Fig. 2b) [6]. Self-priming is a unique mechanism that instead of using a tRNA to prime reverse transcription relies on a 5 ' segment of the mRNA from an LTR-retrotransposon to function as the primer. This mechanism was first demonstrated to occur for the LTR-retrotransposon Tf1 of $S$. pombe but has since been implicated in LTR-retrotransposons in hosts from fungi to vertebrates [13-19].

We decided to test Tj1 for transposition activity because it was one of the families of transposable elements that clustered in the centromeres of $S$. japonicus and determining insertion sites produced de novo would provide information about whether its integration occurred in centromere associated sequences. By expressing Tj1 marked with nеo (Tj1-nеo) in Schizosaccharomyces pombe we found the element was functional and readily produced integration events in chromosomal sites. The sequences of integration sites showed that $\mathrm{Tj} 1$ integrates upstream of RNA pol III transcribed genes. In the case of tRNA genes the inserts occurred between 1 and $10 \mathrm{bp}$ upstream of predicted transcription start sites (TSSs). This integration bias together with the clusters of tRNA genes in the centromeres of $S$. japonicus indicates the clusters of $\mathrm{Tj} 1$ in centromere sequences resulted from the integration preference of $\mathrm{Tj} 1$.

\section{Results}

One possible reason why there are clusters of LTRretrotransposons in the centromeres of S. japonicus is that integration was targeted to these sequences. In order to test this possibility an active retrotransposon must be identified and de novo integration events mapped. To determine whether $\mathrm{Tj} 1$ possesses transposition activity we developed a genetic assay to detect Tj1 integration in $S$. pombe. We inserted neo upstream of the polypurine tract (PPT) in a full-length copy of $\mathrm{Tj} 1$ that was present in a high copy plasmid (Tj1-neo). We also constructed a version of this plasmid that removed the stop codon from the 3 ' end of gag, resulting is one long ORF encoding all Tj1 proteins (Tj1-neo TGAx). A third plasmid was produced that expressed $\mathrm{Tj} 1-n e o$ from the $n m t 1$ promoter located on a plasmid (Nmt1-Tj1-neo). To detect transposition events S. pombe patches expressing the three versions of $\mathrm{Tj} 1-n e o$ were replica printed to medium containing 5-fluoroorotic acid (5FOA). This compound selects against the presence 
a

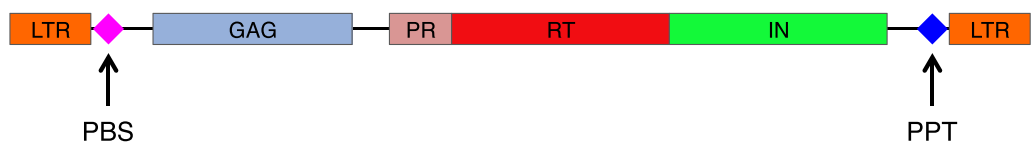

b
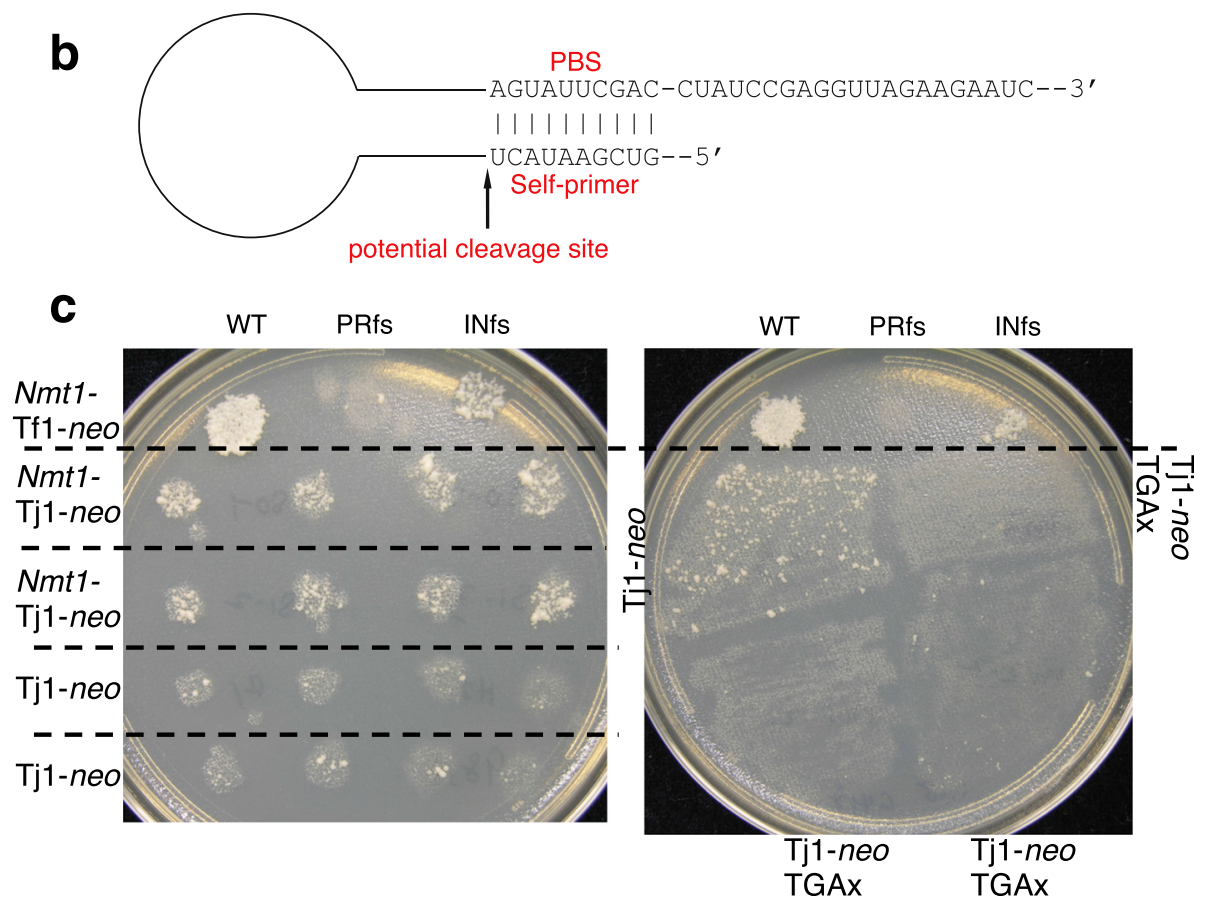

Fig. 2 Tj1 is an active LTR-retrotransposon and likely relies of self-priming to initiate reverse transcription. a The structure of Tj1 is shown. Indicated are the positions of LTRs, and the coding sequences for Gag, PR, RT, and IN. The primer binding site (PBS) and the polypurine tract (PPT) are shown. $\mathbf{b}$ Sequence from the 5' LTR is shown indicating the upstream region that has complementarity to the PBS. We propose this serves as a self-primer of reverse transcription. Also shown is the potential cleavage site that is necessary to initiate priming of reverse transcription. c The FOA/G418 plates from the transposition assay. Left Panel: the top row shows transposition activity of Nmt1-Tf1-neo controls expressing wild type Tf1-neo, Tf1-neo with the protease frameshift (PRfs), and Tf1-neo with the integrase frameshift (INfs). Rows 2 and 3 each contain 4 independent transformants of Nmt1-Tj1-neo. Rows 4 and 5 each contain 4 transformants of Tj1-neo expressed from the Tj1 promoter. Growth of the patches is a measure of transposition frequency. Right Panel: The top row contains the Nmt1-Tf1-neo controls. In the second row the patch on the left contains Tj1-neo. The patch on the right side of the second row and the two patches on the $3^{\text {rd }}$ row are three independent transformants of Tj1-neo with a mutation that removes the stop codon at the end of Gag and as a result fuses all coding sequence into one ORF (Tf1-neo TGAx)

of the $U R A 3$ gene in the expression plasmid. Cells lacking the Tj1-neo present in the plasmid must have integration events to be resistant to G418. In the final step of the assay the patches were replica printed to media containing 5FOA and G418 to test for the presence of integrated copies of Tj1-neo (Fig. 2c). As a control for positive transposition we included cells expressing Nmt1-Tf1-neo, a highly active LTR-retrotransposon originally isolated from $S$. pombe [20-22]. The transposition assay of Nmt1-Tf1-neo produced high levels of resistance to G418 as previously described $[15,21,22]$. For a no transposition control we used Nmt1-Tf1-neo with a frame shift mutation at the N-terminus of IN (Tf1-neo INfs). This greatly reduced G418 resistance indicating as previously reported that the majority of the resistance produced by wild type Nmt1-Tf1-neo was due to integration events [15, 21, 22].
A frame shift in the $\mathrm{N}$ terminus of PR in Nmt1-Tf1-neo (Tf1-neo PRfs) produced little or no G418 resistance demonstrating that translation of $\mathrm{PR}$ and $\mathrm{RT}$ was required for the residual IN independent resistance of Tf1-neo INfs caused by homologous recombination between cDNA and pre-existing Tf sequences. Importantly, expression of Nmt1-Tj1-neo produced moderate levels of G418 resistance in eight independent transformants of $S$. pombe (Fig. 2c, left Panel). Cells containing Tj1-neo showed lower levels of G418 resistance, indicating transposition frequencies were lower when $\mathrm{Tj} 1$ was expressed from its own promoter (Fig. 2c, left Panel). Cells expressing Tj1-neo TGAx showed virtually no resistance to G418, indicating that $\mathrm{Tj} 1$ proteins expressed as a single primary translation product were inactive. The G418 resistance of Nmt1-Tj1-neo and Tj1-neo indicated that Tj1 was 


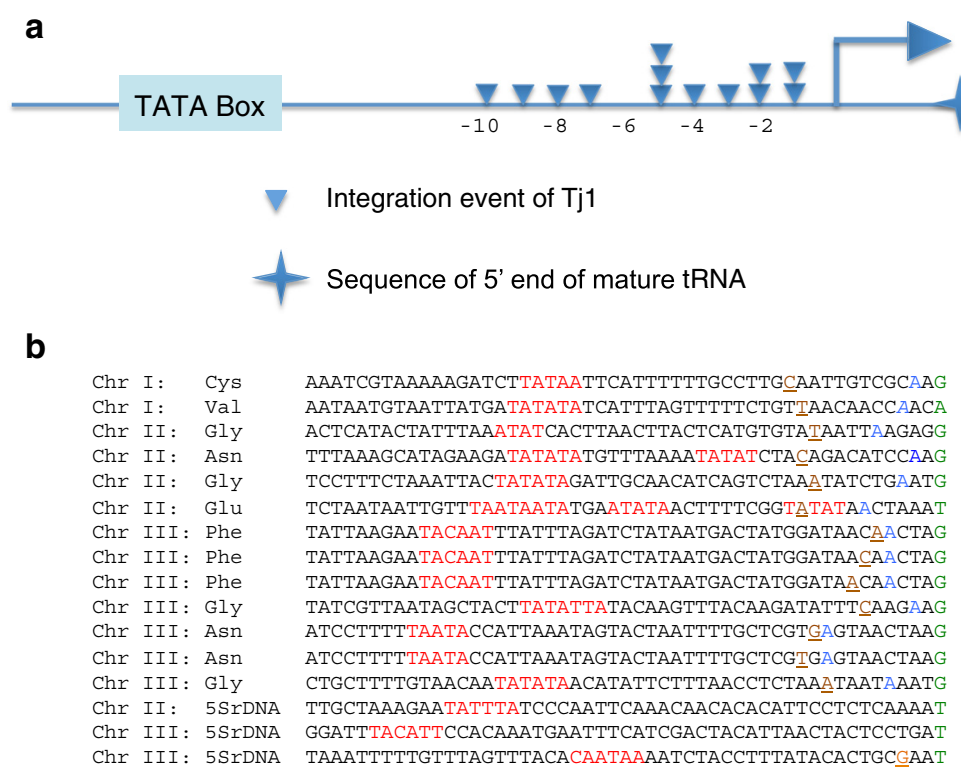

Fig. 3 The positions of Tj1 integration clustered upstream of the TSSs of RNA pol III transcribed genes. a The region upstream of tRNA genes is diagramed showing the nucleotide positions of Tj1 integration. The TATA box is represented and the TSS is symbolized by the right-facing arrow. The star represents the sequence of the $5^{\prime}$ end of the mature RNA species. While the linear representation is not drawn to scale the exact nucleotide positions of each integration site relative to the TSSs is shown. Some integrations mapped to the same tRNA gene but most occurred at different genes (Table 1). The two integrations downstream of the TSSS of $5 S$ rRNA genes are not shown. $\mathbf{b}$ Sequences of the integration sites (Brown underlined) are shown upstream of the RNA pol III transcribed genes. Also shown are the putative TATA boxes (Red), the predicted TSSs (blue) and the 5' nucleotide encoding the 5' end of the mature RNA species (Green)

capable of generating de novo integration events. The lack of activity of TGAx indicated that similar to Type C retroviruses, Tj1 function requires a stop codon at the end of Gag.

We determined the sequences of insertion sites by isolating genomic DNA from patches of $G 418^{\mathrm{R}}$ cells produced by Nmt1-Tj1-neo. The DNA was fragmented by digestion with $M s p \mathrm{I}$, ligated to a linker, and then insertion sites were amplified by PCR using primers specific for the Tj1 LTR and the linker (Methods). The sequences of PCR products identified 17 unique positions where Tj1 integrated (Table 1). We found 13 of the 17 integration sites

Table 1 Position of Tj1 integration in S. pombe

\begin{tabular}{|c|c|c|c|c|c|}
\hline Type & Gene Name & No. of inserts & Distance from sequence of mature RNA & Distance from transcription initiation site of RNA ${ }^{a}$ & Orientation \\
\hline tRNA & SPATRNAVAL.01 & 1 & -11 & -8 & Forward \\
\hline tRNA & SPATRNACYS.02 & 1 & -11 & -10 & Forward \\
\hline Gene & SPAC12B10.11 ${ }^{\mathrm{b}}$ & 1 & 1018 & NA & Reverse \\
\hline tRNA & SPBTRNAGLY.03 & 1 & -10 & -5 & Forward \\
\hline 5S rRNA & SPRRNA.27 & 1 & 41 & NA & Forward \\
\hline tRNA & SPBTRNAASN.02 & 1 & -11 & -9 & Forward \\
\hline tRNA & SPBTRNAGLY.09 & 1 & -10 & -7 & Forward \\
\hline tRNA & SPBTRNAGLU.08 & 1 & -11 & -5 & Forward \\
\hline tRNA & SPCTRNAPHE.04 & 3 & $-5,-6,-7$ & $-1,-2,-3$ & Forward \\
\hline tRNA & SPCTRNAGLY.11 & 1 & -6 & -4 & Forward \\
\hline $5 S$ rRNA & SPRRNA.26 & 1 & 30 & NA & Forward \\
\hline tRNA & SPCTRNAASN.05 & 2 & $-11,10$ & $-1,-2$ & Forward \\
\hline $5 S$ rRNA & SPRRNA.06 & 1 & -3 & NA & Forward \\
\hline tRNA & SPCTRNAGLY.12 & 1 & -9 & -5 & Forward \\
\hline
\end{tabular}


occurred immediately upstream of tRNA genes. Three other integrations were near the $5^{\prime}$ end of 5S RNA genes showing that $\mathrm{Tj} 1 \mathrm{had}$ a strong preference for integration upstream of RNA pol III transcribed genes. One integration event occurred in the coding sequence of exg2, an RNA pol II transcribed gene. The integration sites upstream of tRNA genes were positioned between 5 and $11 \mathrm{bp}$ upstream of the sequence encoding the mature $5^{\prime}$ ends of the RNAs (Table 1). However, the integrations at the 5S RNA genes were more broadly distributed and were located between $3 \mathrm{bp}$ upstream to $41 \mathrm{bp}$ downstream of the sequence encoding the mature $5^{\prime}$ end of the RNAs (Table 1).

To map where integration occurred relative to RNA pol III transcription initiation we used positions of upstream TATA boxes to predict the locations of transcription initiation. Unlike the tRNA genes of $S$. cerevisiae, humans, and many other species, S. pombe tRNA genes have upstream TATA boxes that recruit TBP to sites where transcription initiates [23]. This analysis indicated that Tj1 integrated as close as 1, 2, and 3 bp upstream of the start of transcription (Fig. 3). The integration furthest from the start of transcription was $10 \mathrm{bp}$ upstream of the TSS.

One surprising feature of all the integration events at the RNA pol III transcribed genes is that the Tj1 elements integrated in the same orientation relative to the target gene such that transcription of $\mathrm{Tj} 1$ was in the same direction as the RNA pol III gene (Table 1).

\section{Discussion}

The identification of an intact LTR-retrotransposon from $S$. japonicus that is capable of inserting its sequence into chromosomal sites of the related fission yeast, S. pombe, offered the first opportunity to study the integration behavior of a transposable element from S. japonicus. Tj1-neo readily generated G418 resistance when expressed in S. pombe. The finding that the insertions had Tj1 LTR sequence that transitioned to $S$. pombe sequence at the first bp after the LTR provided strong evidence that Tj1-neo was active and capable of generating bona fide integration events. Our assays also revealed that the expression of Tj1 is similar to Type C retroviruses in that a stop codon at the end of Gag must be present for efficient integration.

Importantly, the positions of Tj1-neo integration showed a strong preference for sequences immediately upstream of RNA Pol III transcribed genes. None of the Pol III transcribed genes with integration were adjacent to centromere sequence indicating that Tj1 recognized Pol III transcription units not centromeres. This integration behavior is strikingly similar to the integration of the Saccharomyces cerevisiae LTR-retrotransposon Ty3 that integrates within a few bp of RNA Pol III transcription start sites [24-30]. The mechanism responsible for this integration has been determined by an elegant series of experiments demonstrating that portions of RNA Pol III transcription factor IIIB subunits, Brf1 and TBP interact with IN and Ty3 cDNA to position integration [31-33]. Whether Tj1 uses a similar mechanism will be the subject of future research. Thus far we were unable to identify amino acid similarities between the INs of Tj1 and Ty3 which could be important for interact with targeting factors.

The finding that $\mathrm{T} j 1$ integrated with the same orientation as the transcription of the RNA pol III transcribed genes is unique. Although a truncation in a subunit of TFIIIC allows just one orientation of Ty3 integration, we know of no wild type LTR retrotransposon or retrovirus other than $\mathrm{Tj} 1$ that integrates with a fixed orientation relative to the transcription of target genes [34]. While this result indicates the interactions between $\mathrm{Tj} 1 \mathrm{IN}$, its target sites, and its CDNA constrain the orientation of integration, it is formally possible that a selection existed that prevented the survival of integration in the opposite orientation.

The integration of Ty3 a few bp upstream of tRNA genes is a targeting mechanism that avoids disrupting coding sequence. In addition, the integration of Ty3 does not damage regulatory elements because the promoters of tRNA genes consist of A box and B box sequences that are within the transcribed sequences of the gene. However, recent studies of tRNA genes have shown in some species of Schizosaccharomyces such as S. pombe and S. octosporus, TATA box sequences are present between positions -20 and -35 bp upstream of the start of transcription [35]. Importantly, in S. japonicus, it appears that tRNA genes do not have upstream TATA boxes, indicating that Tj1 integration would not integrate between the promoter elements and the TSSs of tRNA genes [35]. Such an insertion would very likely disrupt the function of the promoters.

It is possible the clustering of LTR-retrotransposons in the centromeres of S. japonicus is the result of selective pressure either resulting from improved fitness of integration in these regions or due to reduced fitness associated with integration outside of centromeres. However, our finding that Tj1 integration occurs a few bp upstream of tRNA genes indicates that, at least in the case of Tj1, the transposable element sequences in the centromeres of $S$. japonicus are the result of the integration mechanism. This finding raises the possibility that integration of transposable elements in centromere regions is more general and that clustering of transposable elements in the centromeres of other eukaryotes could also be the result of integration bias. This possibility is supported by the observation that the LTR-retrotransposon Tal1 integrates with a strong bias for centromeric repeats of Arabidopsis thaliana [36]. Other evidence suggesting that integration into centromere sequences is more general includes the chromovirus lineage of LTR-retrotransposons [37-39]. The INs of these elements contain a chromodomain (CHD) that 
in proteins such as HP1 interacts with histone H3 methylK9 [40, 41]. When CHDs were identified in retrotransposon INs, it was suggested they play a role in target specificity $[42,43]$. Subsequent experiments with three groups of chromoviruses showed that the CHDs of their INs direct subnuclear localization to foci coincident with heterochromatin [44]. The chromodomain of the fungal element, MAGGY, interacts with histone H3 dimethyland trimethyl-K9, and when the MAGGY chromodomain is fused to the IN of the Tf1 retrotransposon, new Tf1 insertions in $S$. pombe are directed to sites of H3 K9 methylation [44].

\section{Conclusions}

Considerable evidence indicates that transposable elements contribute to the function of centromeres. Mutations that reactivate transposable elements in centromeres cause loss of heterochromatin which results is reduced binding of cohesin. This leads to reduced sister chromatid cohesion in $S$. pombe [45], loss of centromere condensation in A. thaliana [46], and chromosome segregation defects during meiosis in the mouse [47-49]. The question of how centromeres form requires an understanding of transposable elements and why they accumulate in centromeres. Although it is possible some transposable elements accumulate in centromere regions as the result of evolutionary selection, evidence is emerging that many transposable elements accumulate in centromeres because of integration patterns. Our finding that Tj1 integrates upstream of tRNA genes represents a unique strategy for concentrating transposable elements in the centromere. Compared with the LTR-retrotransposons that use CHDs to target centromeric heterochromatin [44], Tj1 integration is an evolutionary convergent method of localizing in centromeres and underscores the importance of the transposable elements in centromeres.

\section{Methods \\ Media}

Agar plates containing EMM were used to grow cells [50]. These plates were supplemented with $2 \mathrm{gm} / \mathrm{l}$ mixtures of an equal weight of all amino acids, 2.5 times more adenine with no uracil. Moreover, a final concentration of $10 \mu \mathrm{M}$ thiamine was used to repress the transcription of $\mathrm{Tj} 1$ driven by the $n m t 1$ promoter. Further, EMM plates were supplemented by $1 \mathrm{~g} / 1$ - fluoroorotic acid (5-FOA) and $50 \mathrm{mg} / \mathrm{mL}$ of uracil to eliminate the plasmid with Tj1-neo. Transposition was measured on plates that contained YES plus $1 \mathrm{~g} / \mathrm{l} 5$-FOA and $0.5 \mathrm{~g} / \mathrm{l} \mathrm{G418}$ as previously described $[15,51]$.

Plasmid construction, sample preparation, and sequencing The sequence of $\mathrm{Tj} 1$ with annotations is available from Genbank with the accession number KT447435. The
Table 2 Plasmids

\begin{tabular}{ll}
\hline Plasmid & Description \\
\hline PHL2848 & Full-length copy of Tj1-neo \\
pHL2851 & $\begin{array}{l}\text { Full-length copy of Tj1-neo, stop codon between gag } \\
\text { and pol removed }\end{array}$ \\
pHL2861 & $\begin{array}{l}\text { Tj1-neo, putative self-priming sequence in 5'-LTR fused } \\
\text { to } n m t 1 \text { promoter }\end{array}$ \\
\hline
\end{tabular}

plasmids used in this study are listed in Table 2. Fulllength Tj1 with neo upstream of the ppt was cloned by PCR from wild type S. japonicus (YHL9763) and inserted into the XhoI and BamHI sites of pHL891 [16] to create pHL2848. pHL2851 was identical to pHL2848 except the stop codon at the $3^{\prime}$ end of gag was removed, resulting in a single ORF encoding all Tj1 proteins. To create pHL2861, the plasmid that expresses Tj1-neo from the $n m t 1$ promoter, we fused the self-priming sequence in the $5^{\prime}$ LTR that complements the PBS to the nmt1 promoter. All Tj1 plasmids were transformed into $S$. pombe YHL912 ( $h^{-}$, leu1-32, ura4-294) for transposition assays.

To generate the library of insertion sites genomic DNA was isolated from G418 resistant patches containing integration events. The genomic DNA was digested by MspI and a linker was ligated to the fragments. The linker ligated DNA fragments were digested with $\mathrm{BamHI}$ to avoid amplifying the 5 ' LTRs. The junction sequence downstream of the 3' LTR was amplified in PCR reactions that contained primers annealing to the LTR and the linker. PCR products were cloned in TOPO vector pCR2.1. These clones were used for Sanger sequencing.

\section{Mapping of Tj1 integration sites on the genome of $S$. japonicus}

The sequence of the LTR and the linker were trimmed and the remaining sequences were used to find the coordinate of the insertion site by BLAST.

\section{Abbreviations \\ 5FOA: 5-fluoroorotic acid; bp: Base pair; CHD: Chromodomain; mg: Milligram; ml: Milliliter; IN: Integrase; LTR: Long terminal repeat; PRfs: Protease frameshift; PBS: Primer binding site; INfs: Integrase frameshift; TSS: Transcription start site. \\ Competing interests \\ The authors declare that they have no competing interests. \\ Authors' contributions \\ YG designed the experiments, prepared the plasmids containing Tj1, isolated integration events, determined the sequences of the insertion sites, and prepared figures. PKS prepared a draft of the manuscript, characterized the insertion sites, and prepared figures. HLL supervised the project, edited the manuscript and prepared figures. All authors have read and approve of the final version of the manuscript.}

\section{Acknowledgements}

This research was supported by the Intramural Research Programs of the $\mathrm{NIH}$ from the Eunice Kennedy Shriver National Institute of Child Health and Human Development. We thank Dr. Nicholas Rhind for his help in accessing sequences of S. japonicus. 


\section{Author details}

${ }^{1}$ Section on Eukaryotic Transposable Elements, Program in Cellular Regulation and Metabolism, Eunice Kennedy Shriver National Institute of Child Health and Human Development, National Institutes of Health, Building 18 T, room 106, Bethesda, MD 20892, USA. ${ }^{2}$ Present address: University of Texas Southwestern Medical Center, Dallas, Texas, USA.

\section{Received: 6 July 2015 Accepted: 22 September 2015} Published online: 09 October 2015

\section{References}

1. Sun X, Wahlstrom J, Karpen G. Molecular structure of a functional Drosophila centromere. Cell. 1997;91:1007-19.

2. Dawe RK. RNA interference, transposons, and the centromere. Plant Cell. 2003;15:297-301.

3. Sun X, Le HD, Wahlstrom JM, Karpen GH. Sequence analysis of a functional Drosophila centromere. Genome Res. 2003;13:182-94.

4. Schueler MG, Sullivan BA. Structural and functional dynamics of human centromeric chromatin. Annu Rev Genomics Hum Genet. 2006;7:301-13.

5. Glockner G, Heidel AJ. Centromere sequence and dynamics in Dictyostelium discoideum. Nucleic Acids Res. 2009;37:1809-16.

6. Rhind N, Chen Z, Yassour M, Thompson DA, Haas BJ, Habib N, et al. Comparative functional genomics of the fission yeasts. Science. 2011;332:930-6.

7. Contreras-Galindo R, Kaplan MH, He S, Contreras-Galindo AC, Gonzalez Hernandez MJ, Kappes F, et al. HIV infection reveals widespread expansion of novel centromeric human endogenous retroviruses. Genome Res. 2013;23:1505-13.

8. Li B, Choulet F, Heng Y, Hao W, Paux E, Liu Z, et al. Wheat centromeric retrotransposons: the new ones take a major role in centromeric structure. Plant J. 2013:73:952-65.

9. Plohl M, Mestrovic N, Mravinac B. Centromere identity from the DNA point of view. Chromosoma. 2014;123:313-25.

10. Gao D, Jiang N, Wing RA, Jiang J, Jackson SA. Transposons play an important role in the evolution and diversification of centromeres among closely related species. Front Plant Sci. 2015;6:216.

11. Slotkin RK, Martienssen R. Transposable elements and the epigenetic regulation of the genome. Nat Rev Genet. 2007:8:272-85

12. Rein A, Levin JG. Readthrough suppression in the mammalian type $C$ retroviruses and what it has taught us. New Biol. 1992:4:283-9.

13. Levin HL. It's prime time for reverse transcriptase. Cell. 1997;88:5-8.

14. Levin HL. An unusual mechanism of self-primed reverse transcription requires the $\mathrm{RNase} H$ domain of reverse transcriptase to cleave an RNA duplex. Mol Cell Biol. 1996;16:5645-54

15. Levin HL. A novel mechanism of self-primed reverse transcription defines a new family of retroelements. Mol Cell Biol. 1995;15:3310-7.

16. Lin JH, Levin HL. A complex structure in the mRNA of Tf1 is recognized and cleaved to generate the primer of reverse transcription. Genes Dev. 1997;11:270-85.

17. Lin JH, Levin HL. Reverse transcription of a self-primed retrotransposon requires an RNA structure similar to the U5-IR stem-loop of retroviruses. Mol Cell Biol. 1998;18:6859-69.

18. Butler M, Goodwin T, Simpson M, Singh M, Poulter R. Vertebrate LTR retrotransposons of the Tf1/Sushi group. J Mol Evol. 2001;52:260-74.

19. SanMiguel P, Tikhonov A, Jin YK, Motchoulskaia N, Zakharov D, Melake-Berhan A, et al. Nested retrotransposons in the intergenic regions of the maize genome [see comments]. Science. 1996;274:765-8.

20. Levin HL, Weaver DC, Boeke JD. Two related families of retrotransposons from Schizosaccharomyces pombe. Mol Cell Biol. 1990;10:6791-8.

21. Levin HL, Boeke JD. Demonstration of retrotransposition of the Tf1 element in fission yeast. EMBO J. 1992;11:1145-53.

22. Levin HL, Weaver DC, Boeke JD. Novel gene expression mechanism in a fission yeast retroelement: Tf1 proteins are derived from a single primary translation product. EMBO J. 1993:12:4885-95.

23. Hamada M, Huang Y, Lowe TM, Maraia RJ. Widespread use of TATA elements in the core promoters for RNA polymerases III, II, and I in fission yeast. Mol Cell Biol. 2001;21:6870-81.

24. Qi X, Daily K, Nguyen K, Wang H, Mayhew D, Rigor P, et al. Retrotransposon profiling of RNA polymerase III initiation sites. Genome Res. 2012;22:681-92.

25. Chalker DL, Sandmeyer SB. Ty3 integrates within the region of RNA polymerase III transcription initiation. Genes Dev. 1992;6:117-28.
26. Sandmeyer SB, Hansen L, Chalker DL. Integration specificity of retrotransposons and retroviruses. Ann Rev Genet. 1990;24:491-518.

27. Hansen LJ, Sandmeyer SB. Characterization of a transpositionally active Ty3 element and identification of the Ty3 integrase protein. J Virol. 1990;64:2599-607.

28. Chalker DL, Sandmeyer SB. Transfer RNA genes are genomic targets for de novo transposition of Ty3. Genetics. 1990;126:837-50.

29. Sandmeyer SB, Bilanchone WW, Clark DJ, Morcos P, Carle GF, Brodeur GM Sigma elements are position-specific for many different yeast tRNA genes. Nucl Acids Res. 1988;16:1499-515.

30. Sandmeyer SB, Olson MV. Insertion of a repetitive element at the same position in the 5'-Flanking Regions of 2 Dissimilar Yeast Transfer-Rna Genes. Proc Natl Acad Sci USA. 1982:79:7674-8.

31. Qi X, Sandmeyer S. In vitro targeting of strand transfer by the Ty3 retroelement integrase. J Biol Chem. 2012;287:18589-95.

32. Yieh $L$, Hatzis $H$, Kassavetis $G$, Sandmeyer SB. Mutational analysis of the transcription factor IIIB-DNA target of Ty3 retroelement integration. J Biol Chem. 2002;277:25920-8.

33. Yieh L, Kassavetis G, Geiduschek EP, Sandmeyer SB. The Brf and TATA-binding protein subunits of the RNA polymerase III transcription factor IIIB mediate position-specific integration of the gypsy-like element, Ty3. J Biol Chem. 2000:275:29800-7.

34. Aye M, Dildine SL, Claypool JA, Jourdain S, Sandmeyer SB. A truncation mutant of the 95 -kilodalton subunit of transcription factor IIIC reveals asymmetry in Ty3 integration. Mol Cell Biol. 2001;21:7839-51.

35. Iben JR, Maraia RJ. tRNAomics: tRNA gene copy number variation and codon use provide bioinformatic evidence of a new anticodon:codon wobble pair in a eukaryote. RNA. 2012;18:1358-72.

36. Tsukahara S, Kawabe A, Kobayashi A, Ito T, Aizu T, Shin-i T, et al. Centromere-targeted de novo integrations of an LTR retrotransposon of Arabidopsis lyrata. Genes Dev. 2012;26:705-13.

37. Marin I, Llorens C. Ty3/Gypsy retrotransposons: description of new Arabidopsis thaliana elements and evolutionary perspectives derived from comparative genomic data. Mol Biol Evol. 2000;17:1040-9.

38. Gorinsek B, Gubensek F, Kordis D. Phylogenomic analysis of chromoviruses. Cytogenet Genome Res. 2005;110:543-52.

39. Gorinsek B, Gubensek F, Kordis D. Evolutionary genomics of chromoviruses in eukaryotes. Mol Biol Evol. 2004;21:781-98.

40. Jacobs SA, Khorasanizadeh S. Structure of HP1 chromodomain bound to a lysine 9-methylated histone H3 tail. Science. 2002;295:2080-3.

41. Nielsen PR, Nietlispach D, Mott HR, Callaghan J, Bannister A, Kouzarides T, et al. Structure of the HP1 chromodomain bound to histone $\mathrm{H} 3$ methylated at lysine 9. Nature. 2002;416:103-7.

42. Koonin EV, Zhou S, Lucchesi JC. The chromo superfamily: new members, duplication of the chromo domain and possible role in delivering transcription regulators to chromatin. Nucleic Acids Res. 1995;23:4229-33.

43. Malik HS, Eickbush TH. Modular evolution of the integrase domain in the Ty3/Gypsy class of LTR retrotransposons. J Virol. 1999;73:5186-90.

44. Gao X, Hou Y, Ebina H, Levin HL, Voytas DF. Chromodomains direct integration of retrotransposons to heterochromatin. Genome Res. 2008;18:359-69.

45. Pidoux AL, Allshire RC. The role of heterochromatin in centromere function. Philos Trans R Soc Lond B Biol Sci. 2005;360:569-79.

46. Lippman Z, Martienssen R. The role of RNA interference in heterochromatic silencing. Nature. 2004;431:364-70.

47. Bourc'his D, Bestor TH. Meiotic catastrophe and retrotransposon reactivation in male germ cells lacking Dnmt3L. Nature. 2004;431:96-9.

48. Peters $\mathrm{AH}, \mathrm{O}^{\prime}$ Carroll $\mathrm{D}$, Scherthan $\mathrm{H}$, Mechtler $\mathrm{K}$, Sauer $\mathrm{S}$, Schofer $\mathrm{C}$, et al. Loss of the Suv39h histone methyltransferases impairs mammalian heterochromatin and genome stability. Cell. 2001;107:323-37.

49. De La Fuente R, Baumann C, Fan T, Schmidtmann A, Dobrinski I, Muegge K. Lsh is required for meiotic chromosome synapsis and retrotransposon silencing in female germ cells. Nat Cell Biol. 2006;8:1448-54.

50. Forsburg SL, Rhind N. Basic methods for fission yeast. Yeast. 2006;23:173-83.

51. Feng G, Leem YE, Levin HL. Transposon integration enhances expression of stress response genes. Nucleic Acids Res. 2013;41:775-89. 